\title{
Gravitational Wave Background from Supermassive Black Holes
}

\author{
Ch. Filloux and J.A. de Freitas Pacheco \\ Université de Nice-Sophia Antipolis - Observatoire de la Côte d'Azur, \\ Laboratoire Cassiopée, UMR 6202 - BP 4229, F-06304 - Nice Cedex 4 - France \\ E-mails: charline.fillouxegmail.com and pacheco@oca.eu \\ F. Durier \\ Max Planck Institute for Extraterrestrial Physics \\ Giessenbachstraße - 85748 Garching - Germany \\ E-mail: fdurier@mpe.mpg.de
}

\section{J.C.N. de Araujo}

Instituto Nacional de Pesquisas Espaciais - Divisão de Astrofísica Avenida dos Astronautas 1758 - S.J. Campos - 12227-010 SP - Brazil

E-mail: jcarlos@das.inpe.br

Cosmological simulations were performed in which the growth of $100 \mathrm{M}_{\odot}$ black hole seeds as well as the evolution of the host galaxy were followed in a self-consistent way. Seeds grow mainly by accretion of baryonic matter and by coalescences occurring after merger events. The gravitational wave background resulting from these coalescences (essentially the ring-down emission) was estimated from the black hole coalescence rate per mass interval as a function of the redshift, which was estimated for the first time based on the present cosmological simulations. Our results indicate that such a background is a "shot-noise" like with frequencies covering a range from $10^{-6} \mathrm{~Hz}$ up to $\mathrm{mHz}$, a region sensitive by LISA. Such a kind of background allows one to individually detect the ringing-down SMBHs. We then estimate the expected detection rate for LISA.

25th Texas Symposium on Relativistic Astrophysics - TEXAS 2010

December 06-10, 2010

Heidelberg, Germany 


\section{Introduction}

The presence of SMBHs in the center of massive galaxies seems to be a well established fact (see, e.g., Ref. [1]) supported by observations of stellar velocity profiles and shifted lines of water masers detected in the core of the host galaxies. Presently, the general belief is that these objects have been formed by the growth of primordial seeds via matter accretion and coalescences resulting from merger episodes involving their hosts. However, the detection of bright SLOAN quasars associated to SMBHs of $10^{9} M_{\odot}$ at $z \sim 6.5$ see, e.g., Ref. [2]), led some authors to propose an alternative evolutionary scenario, in which these objects would have been formed directly via the gravitational collapse.

Processes leading to the formation of SMBHs release, in particular when coalescences occur, an important amount of gravitational radiation. The evolution of primordial massive stars [3] may lead to the formation of BH seeds having masses in the range $100-500 M_{\odot}$. The newly formed BHs are likely to be distorted with respect to the quiescent Kerr geometry, emitting gravitational waves (GWs), the so-called ring-down radiation.

More massive $\mathrm{BHs}$ in a process of merging emit GWs during the inspiral and the plunge phases and lately, when the two horizons merge. In this case, the GW signal has frequencies that are accessible by LISA.

Coalescences of SMBHs are certainly one of the primary sources of GWs for LISA and many investigations have been devoted to this subject aiming, in particular, to describe in detail the inspiral phase as well as the plunge in order to obtain adequate templates for the waveform [4] and to estimate the expected rate of events. These estimates require not only a previous knowledge of the merging rate at different redshifts, but also of the evolution of the BH mass distribution. Early studies considered that the coalescence rate of $\mathrm{BH}$ binaries is related to the overall merger rate of galaxies, displaying a broad maximum around $\mathrm{z}=3$. Crude estimates based on this picture indicate a total coalescence rate ranging from one up to a hundred events per year [5, 6].

In the present study, cosmological simulations were performed in which the growth of $100 M_{\odot}$ BHs seeds as well as the evolution of the host galaxy were followed in a self-consistent way. Seeds grow mainly by accretion of baryonic matter and by coalescences occurring after merger events, forming as a result SMBHs of up $10^{9} M_{\odot}$.

We here focus essentially on the ring-down emission and investigate if a GW background is formed by the SMBHs. Since this background is shot noise like the ringing-down SMBHs can be in principle individually detected. We then estimate the expected detection rate for LISA.

This paper is organized as follows: in Section 2 the simulations are briefly described, in Sections 3 we study the putative background, the expected detection rate for LISA is given in Section 4, and finally, in Section 5 the conclusions are presented.

\section{The simulations and the SMBH merger rates}

General aspects of the code and results concerning the main correlations between the $\mathrm{BH}$ mass and the properties of the host galaxy were already reported in Ref. [7]. Here, for the sake of completeness, we give only the main characteristics of the code. 
Simulations were performed by using the parallel TreePM-SPM code GADGET-2 in a formulation, despite the use of fully adaptive smoothed particle hydrodynamics (SPM), that conserves energy and entropy [8]. Different physical mechanisms affecting the gas dynamics were introduced, such as cooling free-free transitions, radiative recombinations, $\mathrm{H}_{2}$ molecular transitions, atomic fine-structure level excitation, Compton interactions with CMB photons, local heating by the UV-radiation of newly formed stars, mechanical energy injected by type II and type Ia supernovae as well as by AGNs and diffusion of heavy elements.

All simulations were performed in a cube with a size of $50 h^{-1} M p c$ with two different mass resolutions for the gas/stellar particles respectively equal to $5.35 \times 10^{8} M_{\odot}$ (LR-runs) and $3.09 \times 10^{8} M_{\odot}$ (MR-runs). In all runs a flat $\Lambda C D M$ cosmology was adopted, characterized by a Hubble parameter $h=0.7$ in units of $H_{0}=100 \mathrm{~km} \mathrm{~s}^{-1} \mathrm{Mpc}^{-1}$, by a "vacuum" energy density parameter $\Omega_{\Lambda}=0.7$ and by a total matter energy density parameter $\Omega_{m}=0.3$. The fraction of baryonic matter was taken as $h^{2} \Omega_{b}=0.0224$ and the normalization of the matter density fluctuation spectrum was taken to be $\sigma_{8}=0.9$. Initial conditions were fixed according to the algorithm COSMICS and all simulations were performed in the redshift interval $60 \geq z \geq 0$.

A total of eight runs were performed ${ }^{1}$. The six LR-runs were performed to test feedback parameters defining the fraction of the energy released by supernovae ( $\mathrm{SNe}$ ) injected into the medium and the geometry of jets associated to the AGN phase. These runs are labeled LR1, LR2 and so on. In the two MR-runs (labeled MR1 and MR2), the injected energy by SNe was fixed respectively equal to $5.0 \times 10^{49} \mathrm{erg}$ for type Ia and $3.0 \times 10^{49} \mathrm{erg}$ for type II and they differ only in the adopted model for the injection energy in the "AGN" phase.

As already mentioned the present paper focuses essentially on: a) the ring-down emission and if a GW background is formed by the SMBHs, and b) the expected detection rate of merging SMBHs for LISA. The main ingredient here is the black hole coalescence rate. In Fig. 1, the coalescence rate per unit of volume and per mass interval $\Psi(M, z)$ (in $M p c^{-3} y r^{-1} M_{\odot}^{-1}$ ) is shown as a function of the resulting $\mathrm{BH}$ mass and for different redshifts derived from our simulations. It is worth noting that the maximum $\mathrm{BH}$ mass resulting from the coalescence process is of the order of $5 \times 10^{9} M_{\odot}$.

\section{The background}

Since there is a considerable amount of SMBH formed via merger, it is worth investigating if a background of GWs, essentially via ring-down emission, is generated. The coalescence rate per unit of volume and per mass interval $\Psi(M, z)$ derived from our simulations permits to compute such a putative background.

To proceed, the following ingredients are necessary to calculate the expected flux and the equivalent density parameter.

The coalescence rate in a given interval of mass and redshift seen by an observer at $z=0$ is

$$
d R=\frac{\Psi(M, z)}{(1+z)} \frac{d V}{d z} d M d z
$$

\footnotetext{
${ }^{1}$ Computations were performed at the Center of Numerical Computation of the Côte d'Azur Observatory (SIGAMM).
} 


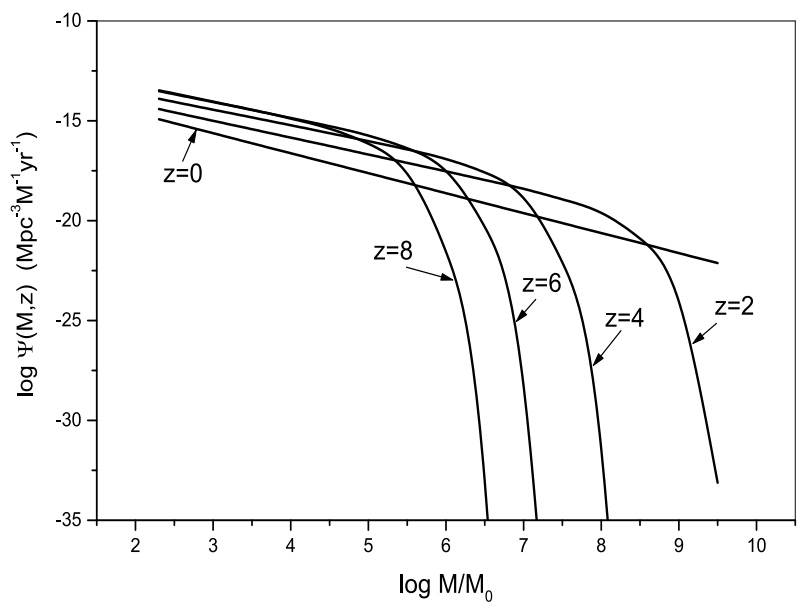

Figure 1: Coalescence rate per unit of volume and per mass interval $\Psi(M, z)$ as a function of the resulting black hole mass. Curves are labeled for different redshifts. For a better visibility in the plot, curves were smoothed.

note that the factor $(1+z)$ that appears in the denominator of the above equation accounts for the time dilation. The comoving volume element $d V$ for a flat cosmology is given by

$$
d V=4 \pi\left(\frac{c}{H_{0}}\right) \frac{r^{2}(z) d z}{\sqrt{\Omega_{\Lambda}+\Omega_{m}(1+z)^{3}}}
$$

and the comoving distance by

$$
r(z)=\frac{c}{H_{0}} \int_{0}^{z} \frac{d x}{\sqrt{\Omega_{\Lambda}+\Omega_{m}(1+x)^{3}}}
$$

The relation between the black hole mass and the observed characteristic "ring-down" frequency is given by [10]

$$
v=\frac{v_{m}}{(1+z)}=\frac{F(a)}{(1+z)}\left(\frac{M_{\odot}}{M}\right) H z
$$

where $a=J c / G M^{2}$ is the spin parameter and the function $F(a)$ is given approximately by [10]

$$
F(a)=1.2 \times 10^{4}\left[\frac{100}{37}-\frac{63}{37}(1-a)^{0.30}\right] \mathrm{Hz}
$$

With the above above equations at hand, it is easy to show that the expected flux is given by:

$$
\phi_{v}=\frac{\varepsilon}{H_{0}}\left(\frac{c}{v}\right)^{3} F^{2}(a) \int_{z_{\min }}^{z_{\max }} \Psi\left(F(a) /\left(1+z^{\prime}\right) v\right) \frac{d z^{\prime}}{\left(1+z^{\prime}\right)^{4} \sqrt{\Omega_{\Lambda}+\Omega_{m}\left(1+z^{\prime}\right)^{3}}} .
$$

The equivalent density parameter reads

$$
\Omega_{g w}(v)=\frac{1}{\rho_{c}} \frac{v \phi_{v}}{c^{3}}
$$




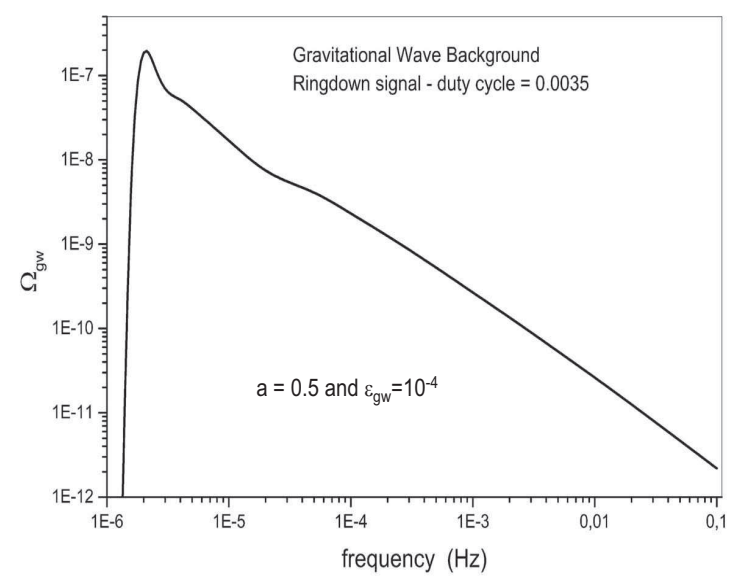

Figure 2: Background of gravitational waves for a ring-down signal for $a=0.5$ and $\varepsilon=10^{-4}$.

Fig. 2 shows the putative background spectrum for $a=0.5$ and $\varepsilon=10^{-4}$. To assess the nature of the background it is necessary to calculate the duty cycle, which relates the time interval between events with their characteristic times. In the present case the duty cycle is $<<1$, which implies that the background is shot-noise like. Therefore, the ringing-down SMBHs can in principle be individually detected. In the next section we consider such an issue.

\section{The expected detection rate for LISA}

Performing a change of variable in Eq. (3.1), i.e., expressing the BH mass in terms of the observed "ring-down" frequency, the expected coalescence rate per logarithm interval of frequency is

$$
\frac{d R}{d \ln (v)}=\int_{z_{\min }(v)}^{z_{\max }(v)} \frac{\Psi(v, z)}{(1+z)}\left|\frac{d M}{d \ln (v)}\right| \frac{d V}{d z} d z
$$

The lower limit $z_{\min }(v)$ appearing in the integral defined by Eq. (4.1) is fixed by the maximum BH mass $M_{b h}(\max )$ present in our simulations. For a given frequency, using Eq. (3.4), the minimum redshift is given by the condition

$$
z_{\min }(v)=\operatorname{Max}\left[0, \frac{1.2 \times 10^{4} F(a)}{v M_{b h}(\max )}\right]
$$

The upper limit $z_{\max }(v)$ represents the redshift below which a gravitational signal can be detected at a given signal-to-noise ratio. The optimal signal-to-noise ratio derived from a matched filtering technique is

$$
\left(\frac{S}{N}\right)^{2}=4 \int_{0}^{\infty} \frac{|\tilde{h}(v)|^{2}}{S_{n}(v)} d v
$$

where $|\tilde{h}(v)|^{2}$ is the spectral density of the signal averaged over both polarizations states and $S_{n}(v)$ is the effective one-sided spectral density of the noise in the detector [9]. 
The "ring-down" waveform is modelled here by a simple damped sinusoidal, i.e.,

$$
h(t)=h_{0} e^{-t / \tau_{m}} \cos \left(\omega_{m} t\right)
$$

where the amplitude $h_{0}$ of the signal is related to the total energy $E$ carried out from the source under the form of gravitational waves by

$$
h_{0}^{2}=\frac{16 G E}{c^{3} d_{L}^{2}} \frac{\tau_{m}}{1+4 Q_{m}^{2}}
$$

where $G$ is the gravitational constant, $d_{L}$ is the luminosity distance and $Q_{m}=\pi v_{m} \tau_{m}=\omega_{m} \tau_{m} / 2$ is the quality factor of the oscillation, given approximately by [10]

$$
Q_{m}=K(a) \simeq 2(1-a)^{9 / 20}
$$

The Fourier transform of the signal is

$$
\tilde{h}(\omega)=\frac{h_{0} \tau_{m}}{\left[1+\left(\omega-\omega_{m}\right)^{2} \tau_{m}^{2}\right]}=h_{0} \tau_{m} f\left(\omega_{m}, \tau_{m}\right)
$$

Notice that $\tilde{h}(v)$ has a Lorentz profile, indicating that the spectral density is peaked around the characteristic frequency $\omega_{m}$.

Using the above equations and assuming that the total energy released under the form of GWs is given by $E=\varepsilon M_{b h} c^{2}$, one obtains for the $S / N$ ratio

$$
\left(\frac{S}{N}\right)=\frac{2.83 \times 10^{-17}}{d_{L}}\left(\frac{\varepsilon M}{M_{\odot}}\right)^{1 / 2} \frac{Q_{m}(a)}{\sqrt{1+4 Q_{m}^{2}(a)}} \frac{1}{\sqrt{v^{2} S_{n}(v)}} .
$$

In Eq. (4.8) the luminosity distance is given in $M p c$ and we have assumed that in the evaluation of the integral in Eq. (4.3), the noise spectral density does not vary considerably near the frequency defining the maximum of the spectral density of the signal. The "ring-down" efficiency $\varepsilon$ was estimated in Ref. [11] to be about $7.8 \times 10^{-4}$ from fully relativistic calculations of head-on collisions between a black hole and a neutron star. In our numerical estimates, a more conservative value equal to $\varepsilon=10^{-4}$ was also considered.

Integration of the coalescence rate $d R / d \ln (v)$ gives the total expected rate of events. Table 1 shows the results for different values of the signal-to-noise ratio, the spin parameter and the "ring-down" efficiency.

Table 1 indicates that the predicted rates are in a very narrow range of values despite the different values of the spin parameter, gravitational wave emission efficiency and $\mathrm{S} / \mathrm{N}$ ratios. In fact, increasing the efficiency or decreasing the $\mathrm{S} / \mathrm{N}$ rate permits to probe effectively a higher volume of the universe.

\section{Conclusions}

Cosmological simulations were performed in which the growth of $100 \mathrm{M}_{\odot} \mathrm{BHs}$ hole seeds as well as the evolution of the host galaxy were followed in a self-consistent way. Seeds grow mainly by accretion of baryonic matter and by coalescences occurring after merger events. 


\begin{tabular}{cccc}
\hline $\mathrm{S} / \mathrm{N}$ & $\mathrm{a}$ & $\varepsilon$ & $\mathrm{R}\left(y r^{-1}\right)$ \\
\hline 5 & 0.50 & $1.0 \times 10^{-4}$ & 15.9 \\
5 & 0.50 & $7.8 \times 10^{-4}$ & 18.0 \\
5 & 0.01 & $1.0 \times 10^{-4}$ & 16.7 \\
10 & 0.50 & $1.0 \times 10^{-4}$ & 14.4 \\
10 & 0.01 & $1.0 \times 10^{-4}$ & 14.8 \\
10 & 0.50 & $7.8 \times 10^{-4}$ & 16.3 \\
\hline
\end{tabular}

Table 1: Coalescence Rates: columns give respectively: (1) the $S / N$ ratio, (2) the spin parameter a, (3) the gravitational wave radiation efficiency $\varepsilon$ and (4) the coalescence rate R.

The GW background resulting from these coalescences (essentially the ring-down emission) was estimated from the BHs coalescence rate, which was estimated for the first time based on the present cosmological simulations.

Our results indicate that such a background is shot-noise like with frequencies covering a region sensitive by LISA. This kind of background allows in principle to individually detect the ring-down SMBHs. For LISA, for example, a total rate of about $15 \mathrm{yr}^{-1}$ is predicted for events having $\mathrm{S} / \mathrm{R}=10$.

\section{Acknowledgments}

JCNA would like to thank CNPq and FAPESP for the financial support.

\section{References}

[1] J. Kormendy and D. Richstone, ARA\&A 33 (1995) 581.

[2] X. Fan, V.K. Narayanan, R.H. Lupton et al., AJ 122 (2001) 2833.

[3] V. Bromm, P.S. Coppi and R.B. Larson, ApJ 527 (1999) L5.

[4] M. Preto, I. Berentzen, P. Berczik, D. Merrit and R. Spurzen, JPhCS 154 (2009) 2049.

[5] M.G. Haehnelt, AIP Conference Proceedings 456 (1998) 45.

[6] D. Merritt and R.D. Ekers, Science 297 (2002) 1310.

[7] Ch. Filloux, F. Durier, J.A. de Freitas Pacheco and J. Silk, IJMPD 19 (2010) 1233.

[8] V. Springel, MNRAS 364 (2005) 1105.

[9] N.J. Cornish, PRD 65 (2001) 022004.

[10] F. Echeverria, PRD 40 (1989) 3194.

[11] F. Loffler, L. Rezzolla and M. Ansorg, PRD 74 (2006) 104018.

[12] S.L. Larson, W.A. Hiscock and R.W. Hellings, PRD 62 (2000) 062001; Online Sensitivity Curve Generator, "http://www.srl.caltech.edu/shane/sensitivity:". 